\title{
QUANTITATIVE STRUCTURE PROPERTY RELATIONSHIP PREDICTION OF ANTOCYANIN CONTENT IN VACUUM DRIED SOUR CHERRY
}

\author{
() Tepić Aleksandra ${ }^{1}$, Šumić Zdravko ${ }^{1}$, Podunavac-Kuzmanović Sanja ${ }^{1}$, \\ Jevrić Lidija1, Gadžurić Slobodan²
}

${ }^{1}$ Faculty of Technology, Department of Applied and Engineering Chemistry, University of Novi Sad, Bul. Cara Lazara 1, 21000 Novi Sad, Serbia

${ }^{2}$ Faculty of Sciences, Department of Chemistry, University of Novi Sad, Trg Dositeja Obradovica 3, 21000 Novi Sad, Serbia

The purpose of the article is to promote and facilitate prediction of antocyanin content in vacuum dried sour cherry. Quantitative structure property relationship (QSPR) analysis was applied on thirteen sorts of cherry. A multiple linear regression (MLR) procedure was used to model the relationships between antocyanin content and antioxidative activity of the investigated sorts of cherry. The complete regression analysis were carried out by PASS 2005, GESS 2006, NCSS Statistical Softwares. The best mathematical model has been developed as a calibration model for predicting the antocyanin content in vacuum dried sour cherry. The quality of the model was validated by leave one out technique as well as by the calculation of statistical parameters for the established model. The established model was used to pre- dict the antocyanin content in vacuum dried sour cherry and close agreement between experimental and predicted values was obtained. The low residual activity and high cross-validated $r^{2}$ values observed $\left(r_{C V}^{2}=0.9124\right)$ indicated the predictive ability of the developed QSPR model. It indicates that the content in vacuum dried sour cherry can be successfully modeled using data of antioxidative activity.

Acknowledgment. These results are part of the projects No.114-451-2373/2011, financially supported by the Provincial Secretariat for Science and Technological Development of Vojvodina and No.172012, supported by the Ministry of Science and Technological Development of the Republic of Serbia.

\section{PREDICTION OF ASCORBIC ACID CONTENT IN TOMATO USING QUANTITATIVE STRUCTURE-RETENTION RELATIONSHIP}

\section{๑) Šumić Zdravko ${ }^{1}$, Tepić Aleksandra ${ }^{1}$, Podunavac-Kuzmanović Sanja ${ }^{1}$, Jevrić Lidija ${ }^{1}$, Gadžurić Slobodan ${ }^{2}$}

${ }^{1}$ Faculty of Technology, Department of Applied and Engineering Chemistry, University of Novi Sad, Bul. Cara Lazara 1, 21000 Novi Sad, Serbia

${ }^{2}$ Faculty of Sciences, Department of Chemistry, University of Novi Sad, Trg Dositeja Obradovica 3, 21000 Novi Sad, Serbia

This article is based on the high performance thinlayer chromatography (HPTLC) analyses of fourteen samples of tomato from different locations in Serbia. A quantitative relationship between the ascorbic acid content in different tomato sorts and the chromatographic retention constants $\left(\mathrm{R}_{M}{ }^{0}\right)$ was investigated by using quantitative structure-retention relationship (QSRR) analyses. The best mathematical model has been developed as a calibration model for predicting the ascorbic acid content in tomato. The quality of the model was validated by leave one out technique as well as by the calculation of statistical parameters for the established model. High values of the correlation coefficients indicate the possibility of applying these model in practice.
Predictive ability of defined mathematical model was tested by comparing and correlating the experimetal and calculated values of ascorbic acid concentration in tomato. Since the correlation was extremly good, theoretical mathematical model can be used to predict the ascorbic acid content in tomato sorts using the HPTLC results.

Acknowledgment. These results are part of the projects No.114-451-2373/2011, financially supported by the Provincial Secretariat for Science and Technological Development of Vojvodina and No.172012, supported by the Ministry of Science and Technological Development of the Republic of Serbia. 